\title{
Posttraumatic Stress Disorder, Quality of Life, and the Subjective Experience in Liver Transplant Recipients
}

Progress in Transplantation 2018, Vol. 28(I) 70-76 (C) 2017, NATCO. All rights reserved. Reprints and permission: sagepub.com/journalsPermissions.nav DOI: I0.II77/I5269248/7746680 journals.sagepub.com/home/pit (S)SAGE

\author{
Georgios Paslakis, MD', Mingo Beckmann, $\mathrm{PhD}^{2}$, \\ Susanne Beckebaum, $\mathbf{M D}^{3}$, Christian Klein, $\mathrm{MD}^{4}$, \\ Jan Gräf, $M D^{5}$, and Yesim Erim, MD'
}

\begin{abstract}
Objective: A high prevalence of posttraumatic stress disorder (PTSD) symptoms among transplant recipients has been associated with a low adherence to treatment and poor survival. It is crucial to detect and prevent the development of posttraumatic stress in transplant settings. Methods: We examined the prevalence of posttraumatic stress symptoms in 3 liver transplant recipients by means of the Essen Trauma Inventory (ETI), a self-report questionnaire. The Short Form-36 was used to assess the perceived health-related quality of life. Patients were asked to indicate the most traumatic events within the context of the liver transplantation procedure. Results: Five patients (4.9\%) fulfilled the criteria for PTSD related to liver disease or transplantation (ETI score greater than 27). In these patients, diagnosis was confirmed by a structured clinical interview. Fourteen (13.6\%) patients had a partial PTSD with the ETI score less than 27 and greater than 16. Posttraumatic stress symptoms were significantly associated with perceived poor physical and mental health-related quality of life. Patients reported that the physicians' disclosure of diagnosis was experienced as traumatic, followed by treatment in an intensive care unit and the liver transplantation itself. Conclusions: The ETI resulted in prevalence rates for PTSD comparable to previous studies in liver transplantation settings. Medical professionals requested additional training in how to deliver severe diagnoses to patients.
\end{abstract}

\section{Keywords}

anxiety, depression, liver transplantation, posttraumatic stress disorder, quality of life

\section{Introduction}

An illness as severe as a chronic liver disease and the liver transplantation procedure itself may be experienced as traumatic events and cause posttraumatic stress-like symptoms or even the full picture of a posttraumatic stress disorder (PTSD) with the core symptoms intrusions, hyperarousal, and avoidance behaviors. The experiences that accompany being diagnosed with serious medical illnesses or their treatment have been shown to be associated with the development of PTSD or subdiagnostic symptoms of PTSD. ${ }^{1}$ A meta-analysis on clinically significant PTSD symptoms after acute coronary syndromes found a prevalence of $16 \%{ }^{2}$ The majority of intensive care patients most likely are not being screened for PTSD despite a higher risk of the syndrome in these patients than in the general population. ${ }^{3}$

A diagnosis of PTSD in the context of transplantation has been investigated predominantly in hematopoietic stem cell transplantation, ${ }^{4}$ with significantly fewer studies on solid organ transplantation. ${ }^{5}$ In the 23 studies reviewed by Davydow et al, ${ }^{6}$ the point prevalence of clinician-ascertained posttransplant PTSD ranged from $1 \%$ to $16 \%$. Consistent predictors of PTSD included history of psychiatric illness prior to transplantation and poor social support posttransplantation. Following heart transplantation and intensive care treatment, Schelling et $\mathrm{al}^{7}$ found an $18 \%$ prevalence of PTSD in a follow-up examination after 6 months. Dew et $\mathrm{al}^{8}$ showed that the heart transplantation was associated with distinct mental distress and posttraumatic symptoms also in caregivers of heart transplant recipients. The occurrence of PTSD in the group of heart transplant recipients has been associated with a reduced adherence to medication, thus increasing the risk of transplant rejection to 14 -fold. ${ }^{9}$ In

\footnotetext{
'Department of Psychosomatic Medicine and Psychotherapy, University Hospital Erlangen, Erlangen, Germany

${ }^{2}$ Department of Psychosomatic Medicine and Psychotherapy, University Hospital Essen, Essen, Germany

${ }^{3}$ Department of Transplant Medicine, University Hospital Münster, Münster, Germany

${ }^{4}$ Department of General, Visceral and Transplantation Surgery, University Hospital Essen, Essen, Germany

${ }^{5}$ Lukaskrankenhaus Neuss, HNO Klinik, Neuss, Germany
}

\section{Corresponding Author:}

Erim Yesim, Department of Psychosomatic Medicine and Psychotherapy, University Hospital Erlangen, Schwabachanlage 6, 91054 Erlangen, Germany.

Email: yesim.erim@uk-erlangen.de 
Table I. Example of Core Posttraumatic Stress Disorder Symptom Items From the Essen Trauma Inventory. ${ }^{\text {a }}$

\begin{tabular}{ll}
$\begin{array}{l}\text { Subscale } \\
\text { Domain }\end{array}$ & Example Question \\
\hline Intrusions & $\begin{array}{l}\text { Did the event cause upsetting thoughts or images that } \\
\text { come to your mind although you don't want them } \\
\text { to? Did you ever suddenly live through the event } \\
\text { again mentally? } \\
\text { Did you try not to think about the event, not to talk } \\
\text { about it, or to suppress feelings about it? Did you try } \\
\text { to avoid situations that remind you of the event (eg, } \\
\text { activities, people, or places)? }\end{array}$ \\
$\begin{array}{l}\text { Did you feel emotionally upset when you were } \\
\text { reminded of the event (eg, helpless, angry, sad, } \\
\text { guilty, embarrassed)? Did you have physical } \\
\text { reactions when you were reminded of the event } \\
\text { (eg, uneasiness, tremor, or racing heartbeat)? }\end{array}$
\end{tabular}

aResponse choices are "not at all," "rarely," "often," or "very often."

the cross-sectional study of lung transplant recipients by Gries et al ${ }^{10}$ clinically significant PTSD symptomatology was observed in $12.6 \%$ of participants.

Rothenhäusler et al described a prevalence of $5 \%$ for PTSD following liver transplantation. ${ }^{11}$ Similarly, Telles-Correia et al observed PTSD rates of $6.4 \%$ among liver recipients. ${ }^{12}$ Annema et $\mathrm{al}^{13}$ examined the prevalence of posttraumatic stress at different time points following liver transplantation and found that the symptoms were more prevalent in the first 5 years after transplantation. Across transplantation studies, risk factors for the development of a PTSD were female gender, low social support, and a history of a psychiatric disorder, as well as the duration of intensive care treatment, the number of complications, and the experience of an acute transplant rejection. ${ }^{11}$

The goal of this study was to investigate the extent to which liver recipients suffer from PTSD using the Essen Trauma Inventory (ETI). ${ }^{14}$ The core items of the ETI are presented in Table 1 . We hypothesized that the easy screening method with the ETI would identify PTSD prevalence by the same rate found in other studies. ${ }^{11,12}$ Furthermore, we took a closer look at those patient experiences in the course of terminal liver disease and transplantation that were reported to having been the most traumatic. The occurrence of PTSD symptoms was hypothesized to be negatively associated with the perceived physical and mental health-related quality of life.

\section{Patients and Methods}

\section{Study Design}

In this study with a cross-sectional design, recipients consulting an outpatient liver transplantation clinic were asked to participate. The study was approved by the local ethics committee. Informed consent was obtained from all participants included in the study.

\section{Population}

The transplant center has approximately 150 candidates on the waiting. The most prominent causes of liver failure were posthepatitis cirrhosis, cirrhosis due to alcoholic liver disease, and cirrhosis due to other reasons. Of the approximately 90 to 100 patients transplanted each year, $57 \%$ to $79 \%$ received an orthotopic liver transplantation, $14 \%$ to $32 \%$ a living donor liver transplantation, and $7 \%$ to $11 \%$ a split liver transplantation.

\section{Setting}

The transplant clinic is located within a large metropolitan city within a large multi-organ transplant center. The clinic follows all transplant recipients beginning with the first posthospital visit. Recipients are seen on a regular basis as part of their postoperative treatment, with consultation intervals ranging from 3 months to once a year depending on the time since transplantation.

\section{Data Collection}

Being a liver recipient was the only inclusion criterion, whereas refusal to participate or the inability to fill in the questionnaires due to language barriers or due to the presence of severe medical complications at the time of consultation were the reasons that did not allow for participation in this study. Thus, over a period of approximately $1 \frac{1}{2}$ years, liver recipients drawn from the posttransplant outpatient clinic were asked to participate.

Data for PTSD were gathered using the $\mathrm{ETI}^{14}$ and for the physical and mental health-related quality of life using the Short Form 36-Item Health Survey (SF-36). ${ }^{15}$ In all patients exceeding predefined cutoff values in the ETI, the structured clinical interview for DSM-IV (SCID) was carried out by phone to certify the diagnosis of a PTSD.

\section{Instruments}

The Essen Trauma Inventory. The ETI is a self-rated questionnaire to assess symptoms of PTSD. The ETI is based on the $D S M-I V$ diagnostic criteria for a PTSD and assesses the socalled A1(life threat) and A2 (intense fear, feeling helpless) criteria, which are prerequisite conditions for a PTSD. The ETI includes 23 items assessing PTSD symptoms rated on a 4-point Likert Scale from not at all (0) to very often (3). The sum scores are calculated by adding the item scores with a range of 0 to 69 . The scale has 3 dimensions: intrusions, hyperarousal, and avoidance, which are the core of PTSD symptoms. The reliability of the subscales ranges from 0.82 (hyperarousal) to 0.87 (intrusions). In addition, patients were asked to relate symptoms to their transplantation experience and to name the event experienced as the most traumatic within the transplantation context. Thus, the presence of PTSD symptoms in the ETI was assessed only with regard to the patient's experience with chronic liver disease and subsequent transplantation.

For the assumption of a full PTSD, the A1 criterion (objective threat to life) and the A2 criterion (subjective appraisal of 
the situation): "Did you think that your life was in danger?," "Did you feel helpless?" or "Did you have strong fear or were you full of horror?") according to the DSM-IV had to be fulfilled and a cutoff value of at least 27 points in the ETI was to be achieved. A partial PTSD according to the ETI was assumed in all cases reaching a minimum of 16 points.

The ETI was developed based on the Posttraumatic Stress Diagnostic Scale ${ }^{16}$ and the revised version of the Impact of Event Scale. ${ }^{17}$ Reliability of the total scale (Cronbach $\alpha=$ $0.95)$, as well as the construct and factorial validity were tested in different samples including healthy, but also mentally and physically ill individuals $(\mathrm{n}=952)$. The sensitivity and specificity of the ETI to the diagnosis of a PTSD were $97.3 \%$ and $98 \%$, respectively. High effect sizes (from 1.2 to 4.3 ) to change sensitivity were found. ${ }^{14}$ The construct validity has been verified by significant correlations with several other trauma scales. ${ }^{18}$ The ETI is a screening instrument developed in Germany but has been translated and is in use in 14 countries.

Short Form 36-Item Health Survey, German version (SF-36). The SF-36 is an internationally established self-assessment questionnaire of psychological, physical, and social aspects of quality of life. ${ }^{15}$ It has a high validity and reliability and has been shown to be highly sensitive to change. It contains 36 items and is divided into 8 domains of physical and mental health: physical functioning, role-physical, bodily pain, general health, vitality, social functioning, role-emotional, and psychological well-being. In addition to the scores of the 8 subscales, a mental score and a physical composite score are generated (range: 0 to $100)$. The raw scores are transformed to a scale with a mean of 50 and a standard deviation (SD) of 10. Study scores were compared to the normal population.

Structured clinical interview for DSM-IV. The SCID allows an economical and valid evaluation and the diagnosis of psychiatric syndromes and disorders as defined by the $D S M-I V$. It is often used in studies as the gold standard in determining accurate clinical diagnoses. In this study, the PTSD module of the SCID was applied when appropriate, as described above. A high reliability for the PTSD module ( $\alpha$ coefficient ranging between 0.78 and 1.0) has been reported in numerous studies. ${ }^{19}$

\section{Statistical Analysis}

Data were analyzed using SPSS (IBM Statistics, Armonk, New York). For descriptive analysis, data were expressed as means and SDs. Depending on sample sizes and scale level, group differences were determined using analysis of variance (ANOVA) followed by Scheffé tests, $t$ tests for independent samples, and one-sample $t$ tests, as appropriate. To analyze the bivariate relationships between the dependent and independent variables, Pearson and Spearman correlations were conducted. For all tests, a significance level of $P<.05$ was predetermined.
Table 2. Demographic Characteristics of the Patient Cohort.

\begin{tabular}{lc}
\hline Variable & Total $\mathrm{N}=103, \mathrm{n}(\%)$ \\
\hline Gender & \\
Male & $63(6 \mathrm{I})$ \\
Mean age in years (SD; range), years & $52.3(\mathrm{II} .6 ;$ I8-77) \\
Family status & $14(14)$ \\
Single & $75(72)$ \\
Married & $10(10)$ \\
Separated/divorced/widowed & $4(4)$ \\
Unknown & \\
Educational status & $4(4)$ \\
Without certificate & $65(63)$ \\
Public school/high school & $33(3 \mathrm{I})$ \\
University qualification/certificate & $1(2)$ \\
Unknown & \\
Employment status & $34(33)$ \\
Employed & $7(7)$ \\
Housewife & $3(3)$ \\
Student & $2(2)$ \\
Unemployed & $57(55)$ \\
Retired &
\end{tabular}

\section{Results}

\section{Demographic Characteristics}

A total of 110 of 187 liver recipients were eligible, agreed to participate in the study, and were enrolled after written informed consent. Reasons for refusal were either lack of interest or the perception of the intimate character of the required information. In the end, 103 full data sets for the ETI and 98 full data sets for the SF-36 were available for analysis. Mean values (M) and SD are presented.

The mean age of the patients was 52.3 years $(\mathrm{SD}=11.6)$, with a range of 18 to 77 years. Of the 103 patients, $61 \%$ were men and $39 \%$ were women. Demographic characteristics are presented in detail in Table 2.

\section{Case History Information}

The mean waiting time from transplantation was 165.1 days $(\mathrm{SD}=242.3)$, ranging from 0 to 1916 days $(\mathrm{n}=96)$. The mean time since transplantation was 5.2 years $(\mathrm{SD}=4.0 ; \mathrm{n}=97)$. In $32 \%$ of all cases, the transplant had been conducted in 2 years preceding this study; in $4 \%$ of all cases, however, the transplant had taken place 15 years ago or longer. Hepatitis-associated cirrhosis was the most frequent diagnosis (36.9\%), followed by alcoholic liver cirrhosis (16.5\%). The mean value for treatment duration in an intensive care unit was 8.38 days $(\mathrm{SD}=11.7)$, with a range from 1 to 56 days $(n=100)$. The mean duration of assisted ventilation $(\mathrm{n}=97)$ was 3.54 days $(\mathrm{SD}=6.4)$.

\section{Nature of the Most Traumatic Event}

Having suffered from a chronic liver disease and having gone through all stages of the transplantation procedure, patients in this study reported the following as having been the most 
Table 3. The Correlation Coefficients of the ETI Among the Composite and Subscale Scores of the SF-36.

\begin{tabular}{|c|c|c|c|c|}
\hline $\begin{array}{l}\text { Correlations } \\
\text { SF-36 (short Form 36-Item Health Survey) }\end{array}$ & \multicolumn{4}{|c|}{ ETI } \\
\hline Mental component summary & $-0.59^{c}$ & $-0.62^{c}$ & $-0.47^{c}$ & $-0.57^{c}$ \\
\hline Physical functioning & $-0.38^{c}$ & $-0.38^{c}$ & $-0.29^{a}$ & $-0.43^{c}$ \\
\hline Role-physical & $-0.38^{c}$ & $-0.35^{c}$ & $-0.36^{c}$ & $-0.37^{c}$ \\
\hline Vitality & $-0.4 I^{c}$ & $-0.43^{c}$ & $-0.3 I^{a}$ & $-0.47^{c}$ \\
\hline Social functioning & $-0.44^{c}$ & $-0.48^{c}$ & $-0.29^{a}$ & $-0.44^{c}$ \\
\hline Role-emotional & $-0.58^{c}$ & $-0.59^{c}$ & $-0.54^{c}$ & $-0.50^{c}$ \\
\hline Mental health & $-0.55^{c}$ & $-0.6 \mathrm{I}^{\mathrm{c}}$ & $-0.37^{c}$ & $-0.59^{c}$ \\
\hline
\end{tabular}

Abbreviation: ETI, Essen Trauma Inventory.

${ }^{a}$ Significant on a level of .0I.

bSignificant on a level of .5 .

'Significant on a level of .001 .

traumatic events: disclosure of diagnosis by their physicians $(\mathrm{n}=29,28.2 \%)$, inpatient treatment in an intensive care unit $(\mathrm{n}=12,11.7 \%)$, and the transplant procedure itself $(\mathrm{n}=10$, $9.7 \%)$. A total of $4.9 \%$ of the patients $(n=5)$ stated that no events were experienced as traumatic.

\section{Al and A2 Criteria for PTSD According to DSM-IV}

The A1 criterion (objective life threat) was fulfilled in all patients. The A2 criterion was met in $\mathrm{n}=96$ patients (93.2\%), fulfilling the prerequisite conditions for the diagnosis of a PTSD according to DSM-IV.

\section{PTSD Symptoms and Prevalence According to the ETI}

The mean ETI total score was $8.5(\mathrm{SD}=8.3 ; \mathrm{n}=103)$. The subscale avoidance showed a mean score of $3.1(\mathrm{SD}=3.3)$, the subscale intrusions a mean score of $2.8(\mathrm{SD}=3.1)$, and the subscale hyperarousal a mean score of $2.6(\mathrm{SD}=2.7)$.

The prevalence of full PTSD was $4.9 \%$. The following subjective experiences were found to be associated with a full PTSD: disclosure of the diagnosis $(\mathrm{n}=4)$ and intensive care treatment following the transplant $(\mathrm{n}=1)$.

Of the $\mathrm{N}=103$ patients, $\mathrm{n}=16(15.5 \%)$ met the criteria for partial PTSD from the ETI scores; however, only $\mathrm{n}=$ $14(13.6 \%)$ were classified as partial PTSD once the DSM$I V$ A2 criterion was assessed. The partial PTSD was associated with the following subjective experiences: disclosure of the diagnosis $(n=4)$, the transplant itself $(n=2)$, worries about organ shortage while being on the transplant waiting list $(\mathrm{n}=1)$, intensive care treatment $(\mathrm{n}=1)$, a symptomatic transitory psychotic syndrome following surgery $(\mathrm{n}=1)$, feelings of helplessness following the transplant $(\mathrm{n}=1)$, hepatitis $\mathrm{B}(\mathrm{n}=1)$, other reasons or not specified $(\mathrm{n}=3)$.

\section{Associations Between ETI Scores and Sociodemographic, as well as General Health Data}

A gender-specific difference was displayed by the results: a significantly higher ETI total score $(t=-2.9 ; P=.005)$ was found in women $(\mathrm{M}=11.4 ; \mathrm{SD}=8.0)$ compared to men $(\mathrm{M}=$ 6.7 ; $\mathrm{SD}=8.1)$; this was the case for all the 3 subscales: intrusions, avoidance, and hyperarousal of the inventory $(P<.05)$. Neither age $(P=.59)$ nor educational level $(P=.16)$ or family status $(P=.65)$ was associated with the ETI total scores.

\section{Health-Related Quality of Life}

The mean value for perceived mental health-related quality of life in the whole patient cohort was $51.03(\mathrm{SD}=10.51)$ and was comparable to the standardized norm $(P=.64, \mathrm{n}=98)$; however, the physical health-related quality of life $(\mathrm{M}=44.32$; $\mathrm{SD}=10.16)$ was significantly reduced compared to the norm sample $(t=-5.74 ; P<.001, \mathrm{n}=98)$.

The perceived physical health-related quality of life showed an age effect could be demonstrated $(r=-2.0 ; P=.045, \mathrm{n}=$ 98 ), in terms that a lower physical health-related quality of life was associated with higher age. Gender had no significant effect in the aspect of physical health-related quality of life, whereas women displayed a significantly lower score in the mental health-related quality of life $(t=2.02 ; P=.047, \mathrm{n}=98)$.

\section{PTSD Categories and Quality of Life}

The composite scores for the perceived physical and mental health-related quality of life were significantly associated with the ETI total scores $(r=-0.28, P \leq .01$ and $r=-0.60, P \leq .001$, respectively). Significant associations were found in all of the 8 subscales of the SF-36. A result matrix is depicted in Table 3.

A univariate ANOVA to the mental health-related quality of life composite score for the groups partial PTSD, full PTSD, and no PTSD has brought up a significant effect $(F=17.07$; 


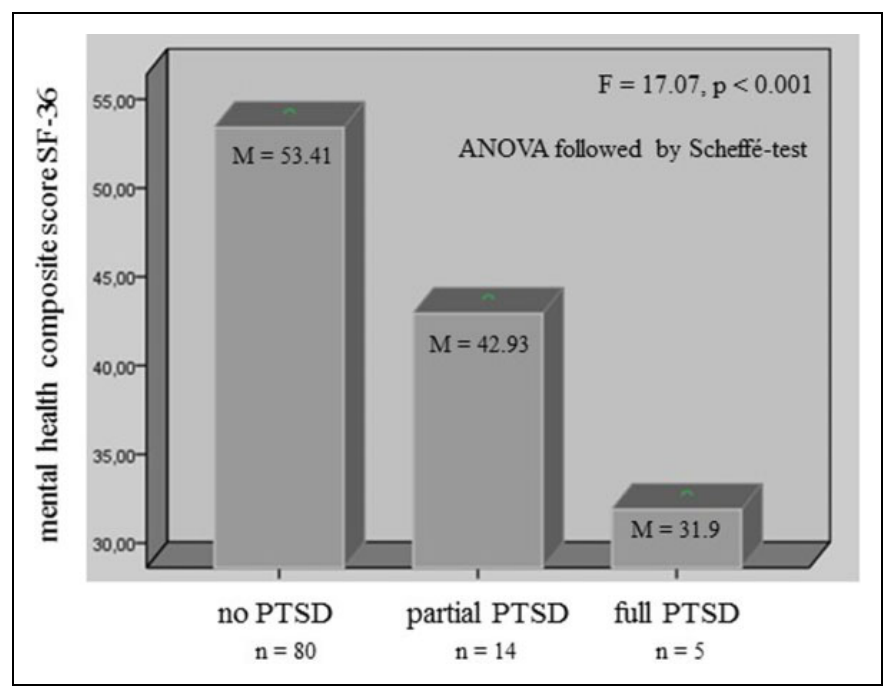

Figure I. Analysis of variance (ANOVA): mental health-related quality of life composite scores in the SF-36 (short Form 36-Item Health Survey) according to posttraumatic stress disorder (PTSD) groups.

$P<.001, \mathrm{n}=98$ ). Post hoc comparisons (Scheffé test) showed significant group differences between no PTSD and partial PTSD, as well as between no PTSD and full PTSD $(P<.001)$, but not between partial PTSD and full PTSD $(P=.11$; Figure 1$)$. By means of a multivariate ANOVA, these differences were found to be significant for each subscale of the SF-36 $(P<.05)$, except for bodily pain (Figure 2$)$. The physical health-related quality of life did not differ significantly between the groups $(F=2.7 ; P=.07)$.

\section{Discussion}

In the present study, we examined the occurrence of PTSD symptoms and a full PTSD and their influence on the quality of life in liver transplant recipients. The PTSD symptoms were only assessed with regard to chronic liver disease and/or liver transplantation.

Showing a prevalence of $5 \%$ for full and $13.6 \%$ for partial PTSD, a total of $18.6 \%$ of recipients suffered from symptoms of a PTSD following transplantation. These results are in line with Rothenhäusler et $\mathrm{al}^{11}$ who described a PTSD prevalence of $4 \%$ for a full and $16 \%$ for a partial PTSD following liver transplantation. In a more recent study, Jin et $\mathrm{a}^{20}$ found a prevalence of $3.7 \%$ for full PTSD and $5.4 \%$ for partial PTSD. Even in the long run, up to 15 years after transplantation, symptoms of posttraumatic stress were found in $10 \%$ of patients, with the highest occurrence of posttraumatic symptoms in the first 5 years after transplantation. Thus, the ETI is an easy screening method and found to lead to prevalence rates that are comparable to previous studies in liver transplant studies. Mintzer et $\mathrm{al}^{21}$ emphasize the underestimation of PTSD prevalence rates in the clinical context of general medicine and in particular with regard to transplantation.

Our result of a $5 \%$ prevalence for postliver transplant PTSD appears to be considerably higher compared to the general

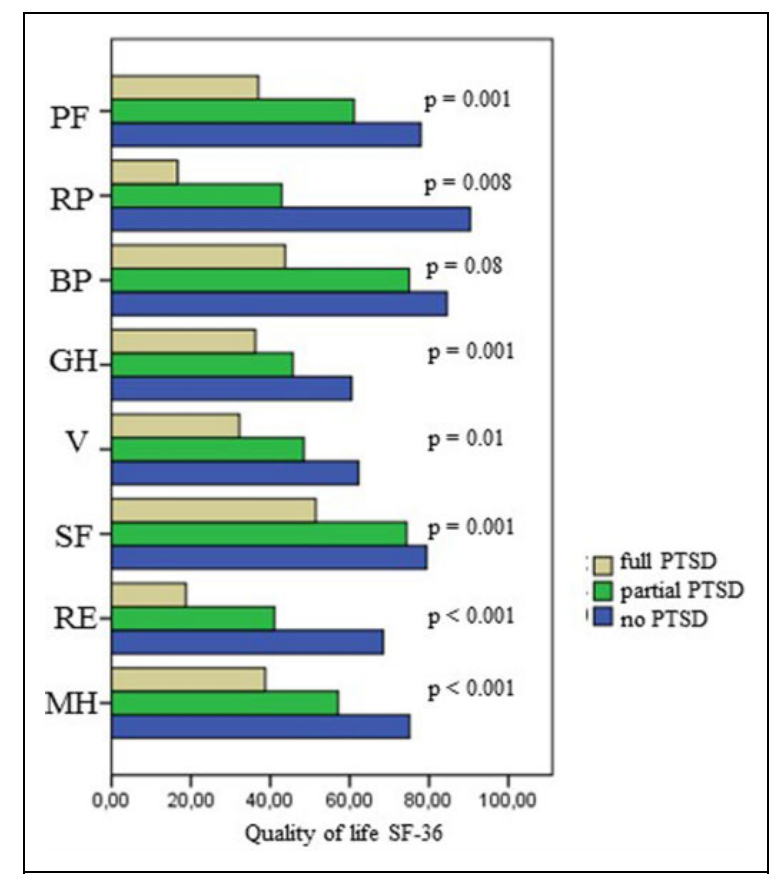

Figure 2. Quality of life dimensions in the SF-36 (short Form 36-Item Health Survey) among posttraumatic stress disorder (PTSD) groups. $\mathrm{BP}$ indicates bodily pain; $\mathrm{GH}$, general health; $\mathrm{MH}$, mental health; $\mathrm{PF}$, physical functioning; RE, role-emotional; RP, role-physical; SF, social functioning; $V$, vitality.

population. Perkonigg et $\mathrm{al}^{22}$ found a point prevalence of $1.6 \%$ for PTSD $-1 \%$ for males and $2.2 \%$ for females. In the present study, women also reached significantly higher scores of PTSD symptoms than men. Women consistently show a prevalence of a PTSD that is 2 times higher than the prevalence in men. ${ }^{23,24}$

There is scarce evidence with respect to the risk factors for the development of a PTSD in the course of a solid organ transplantation. The duration of intensive care treatment, the number of medical complications, and the occurrence of acute rejection have been identified as risk factors that may lead to a PTSD. ${ }^{11}$ Other risk factors for the development of a PTSD were higher model for end-stage liver disease scores and posttransplant complications, while a higher education was a protective factor in the study by Jin et al. ${ }^{20}$ A preexisting psychiatric morbidity, retransplantation, chronic benzodiazepine consumption, age, the type of transplantation, viral hepatitis, and the number of side effects due to immunosuppressants medication were also found to be associated with posttraumatic stress symptoms in patients having undergone solid organ transplantation. ${ }^{5,13}$

Disclosure of the diagnosis, followed by posttransplantation treatment in an intensive care unit, as well as the procedure itself were reported to be the most traumatic events in the present investigation. It seems that disclosing a diagnosis and setting the indication for a transplant is a crucial moment in a patient's life, seriously disrupting their life plans. Similarly, the notification of the need for a heart transplant to patients has been described as a distinct turning point in a patients' life. ${ }^{25}$ In 
a study with heart transplant recipients, $61.2 \%$ of patients had felt the disclosure of the diagnosis to be unexpected and shocking. Patients often report that their doctors' notifications are brief and unemotional. ${ }^{26}$ Thus, top priority must be given to the way of communicating severe diagnoses and procedures in order to facilitate coping. Health professionals trained in communication skills are asked to deliver delicate information in a manner that is not disruptive or traumatic.

The sequelae of PTSD for impaired quality of life have been emphasized in numerous studies. ${ }^{27,28}$ The occurrence of PTSD following a liver transplantation depends on a variety of factors that cannot be foreseen and controlled for; thus, there are no specific measures that may be undertaken in order to minimize the risk. As shown here, a substantial number of liver transplant recipients have fulfilled the criteria of a full PTSD that was related to the transplant-associated procedures. These patients had not received the appropriate mental health care, leading to the assumption that PTSD is underrecognized by medical professionals within the transplant setting. Thus, it is very important that these patients are identified and offered education, counseling, or referrals for mental health evaluation.

Liver transplant recipients attend regular medical visits that offer the opportunity for distribution of self-report screening instruments like the ETI. As posttraumatic stress symptoms may be transitory during the first month following a distressing procedure such as the transplant, screening for PTSD should be performed at least once, for example, at 6 months after the procedure. Completed screens are to be reviewed by the physician, nurses, or a mental health consultant, in order to identify patients who are experiencing posttrauma reactions. Next, these patients may be referred to specialized PTSD treatment for further evaluation and treatment. Due to the fact that some patients refuse referral to mental health care, it is crucial to normalize the idea of treatment.

The greatest limitation of the study lies in the patient population selection and the fact of a single phone interview for ascertaining PTSD. The individuals included are heterogeneous regarding their time on the waiting list and timing of the interview differed in relation to the transplantation itself. We were not able to perform regression analysis due to the small number of patients with a full PTSD. Finally, the ETI is not geared to DSM-V; it was constructed based on the DSM-IV criteria for PTSD.

The occurrence of PTSD in transplant patients is a relevant medical issue. Treatment in the intensive care unit contributes to PTSD, and the presence has a major influence on quality of life posttransplant. In the present article, only liver disease-/ transplantation-related PTSD was assessed in patients within the context of liver transplantation. We found that disclosure of the diagnosis by physicians is salient for the development of PTSD in these patients. Thus, training for medical professionals in communicating diagnoses is important. The prevalence rates for PTSD by means of the ETI were comparable to those found in other studies within the context of liver transplantation.

\section{Declaration of Conflicting Interests}

The author(s) declared no potential conflicts of interest with respect to the research, authorship, and/or publication of this article.

\section{Funding}

The author(s) received no financial support for the research, authorship, and/or publication of this article.

\section{References}

1. Cavalcanti-Ribeiro P, Andrade-Nascimento M, Morais-de-Jesus M, et al. Post-traumatic stress disorder as a comorbidity: impact on disease outcomes. Expert Rev Neurother. 2012;12(8): 1023-1037.

2. Edmondson D, Richardson S, Falzon L, Davidson KW, Mills MA, Neria Y. Posttraumatic stress disorder prevalence and risk of recurrence in acute coronary syndrome patients: a metaanalytic review. PLoS One. 2012;7(6): e38915. doi:10.1371/ journal.pone.0038915.

3. Warlan H, Howland L, Connelly C. Detection of posttraumatic stress symptoms in patients after discharge from intensive care. Am J Crit Care. 2016;25(6):509-515.

4. Esser P, Kuba K, Scherwath A, et al. Posttraumatic stress disorder symptomatology in the course of allogeneic HSCT: a prospective study. J Cancer Surviv. 2017;11(2):203-210. doi:10. 1007/s11764-016-0579-7.

5. Baranyi A, Krauseneck T, Rothenhäusler HB. Posttraumatic stress symptoms after solid-organ transplantation: preoperative risk factors and the impact on health-related quality of life and life satisfaction. Health Qual Life Outcomes. 2013;11:111. doi: 10.1186/1477-7525-11-111.

6. Davydow DS, Lease ED, Reyes JD. Posttraumatic stress disorder in organ transplant recipients: a systematic review. Gen Hosp Psychiatry. 2015;37(5):387-398. doi:10.1016/j.genhoppsych. 2015.05.005.

7. Schelling G, Richter M, Roozendaal B, et al. Exposure to high stress in the intensive care unit may have negative effects on health-related quality-of-life outcomes after cardiac surgery. Crit Care Med. 2003;31(7):1971-1980.

8. Dew MA, Myaskovsky L, DiMartini AF, Switzer GE, Schulberg $\mathrm{HC}$, Kormos RL. Onset, timing and risk for depression and anxiety in family caregivers to heart transplant recipients. Psychol Med. 2004;34(6):1065-1082.

9. Dew MA, Kormos RL, Roth LH, Murali S, DiMartini A, Griffith BP. Early post-transplant medical compliance and mental health predict physical morbidity and mortality one to three years after heart transplantation. J Heart Lung Transplant. 1999:18(6): 549-562.

10. Gries CJ, Dew MA, Curtis JR, et al. Nature and correlates of post-traumatic stress symptomatology in lung transplant recipients. J Heart Lung Transplant. 2013;32(5):525-532. doi:10. 1016/j.healun.2013.01.1046.

11. Rothenhäusler HB, Ehrentraut S, Kapfhammer HP, et al. Psychiatric and psychosocial outcome of orthotopic liver transplantation. Psychother Psychosom. 2002;71(5):285-297.

12. Telles-Correia D, Barbosa A, Mega I, Mateus E, Monteiro E. When does quality of life improve after liver transplantation? A 
longitudinal prospective study. Transplant Proc. 2009;41(3): 904-905. doi:10.1016/j.transproceed.2009.01.051.

13. Annema C, Roodbol PF, Stewart RE, Porte RJ, Ranchor AV. Prevalence of psychological problems and associated transplantrelated variables at different time periods after liver transplantation. Liver Transpl. 2015;21(4):524-538. doi:10.1002/lt.24075.

14. Tagay S, Senf W. Essener Trauma-Inventar (ETI). Manual. Hogrefe, Göttingen; 2014.

15. Bullinger M, Kirchberger I. The SF-36 Questionnaire. Göttingen, Germany: Hogrefe; 1998.

16. Foa E. The Post Traumatic Diagnostic Scale Manual. Minneapolis: NCS; 1995.

17. Weiss DS, Marmar CR. The impact of event scale-revised. In: Wilson JP, Keane TM, eds. Assessing Psychological Trauma and PTSD. New York, NY: Guilford; 1996:399-411.

18. Tagay S, Zararsiz R, Erim Y, et al. Traumatic events and posttraumatic stress disorder in Turkish-speaking patients in primary care. Psychother Psychosom Med Psychol. 2008;58(3-4): 155-161. doi:10.1055/s-2008-1067357.

19. Zanarini MC, Frankenburg FR. Attainment and maintenance of reliability of axis I and II disorders over the course of a longitudinal study. Compr Psychiatry. 2001;42(5):369-374.

20. Jin SG, Yan LN, Xiang B, et al. Posttraumatic stress disorder after liver transplantation. Hepatobiliary Pancreat Dis Int. 2012; 11(1):28-33.

21. Mintzer LL, Stuber ML, Seacord D, Castaneda M, Mesrkhani V, Glover D. Traumatic stress symptoms in adolescent organ transplant recipients. Pediatrics. 2005;115(6):1640-1644.
22. Perkonigg A, Kessler RC, Storz S, Wittchen HU. Traumatic events and post-traumatic stress disorder in the community: prevalence, risk factors and comorbidity. Acta Psychiatr Scand. 2000;101(1):46-59.

23. Tagay S, Herpertz S, Langkafel M, Senf W. Posttraumatic stress disorder in a psychosomatic outpatient clinic. Gender effects, psychosocial functioning, sense of coherence, and service utilization. J Psychosom Res. 2005; 58(5):439-446.

24. Simmons CA, Granvold DK. A cognitive model to explain gender differences in rate of PTSD diagnosis. Brief Treatment Crisis Intervention. 2005;5(3):290-299.

25. Bunzel B, Titscher G, Grundböck A, Wollenek G. "You need a new heart". The problem of diagnostic disclosure from the viewpoint of the affected cardiologic patient. Psychother Psychosom Med Psychol. 1991;41(11):419-428.

26. Lerman C, Daly M, Walsh WP, et al. Communication between patients with breast cancer and health care providers. Determinants and implications. Cancer. 1993;72(9): 2612-2620

27. Supelana C, Annunziato RA, Kaplan D, Helcer J, Stuber ML, Shemesh E. PTSD in solid organ transplant recipients: current understanding and future implications. Pediatr Transplant. 2016;20(1):23-33. doi:10.1111/petr.12628.

28. Schnurr PP, Hayes AF, Lunney CA, et al. Longitudinal analysis of the relationship between symptoms and quality of life in veterans treated for posttraumatic stress disorder. J Consult Clin Psycol. 2006;74(4):707-713. 